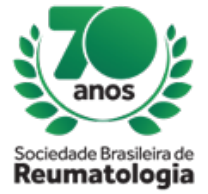

\title{
ANTI-TUMOR NECROSIS FACTOR - ALPHA THERAPY IN A PATIENT WITH CD4 LYMPHOPENIC
} SARCOIDOSIS PHENOTYPE

Marcus Villander Barros de Oliveira Sá (Real Hospital Português de Beneficência, Recife, PE, Brasil), Tamara Cristina Silva Sousa (Real Hospital Portugues de Beneficência, Recife, PE, Brasil), Francisco Barretto (Real Hospital Portugues de Beneficencia, Recife, PE, Brasil), Silverio José Correia Cunha (Real Hospital Portugues de Beneficencia, recife, PE, Brasil), Flavio José Siqueira Pacheco (Real Hospital Portugues de Beneficência, Recife, PE, Brasil)

\section{BACKGROUND}

Sarcoidosis is a systemic inflammatory disease of unknown cause characterized by noncaseating granulomatous inflammation. Lymphopenic sarcoidosis phenotype is a common presentation, can occurs in over $50 \%$ of sarcoidosis patients and is associated with chronic disease. Evidenced-based treatment do not exist for most presentations and there are no data about treatment of lymphopenic sarcoidosis in randomized controled trials. Immunosuppressive agents like corticosteroids, leflunomide, azathioprine, mycophenolate mofetil, methotrexate, and anti-TNF- $\alpha$ can be used for sarcoidosis treatment and potentially cause adverse events, especially infections and lymphopenia.

\section{CASE REPORT}

47 years old woman, with dyspnea, cough, arthralgia and fatigue. No fever, weight loss, diarrhea or jaundice were reported. No history of occupational exposures, drug abuse or recurrent infections. Computed tomography (CT) of the chest showed interstitial micronodules with perilymphatic distribution in both lungs and discrete lymph node enlargement in mediastinum. Lung and lymph nodes were biopsied and showed chronic noncaseating granulomatous inflammatory process. Bacterial, mycobacteria, fungi and cancer etiology were ruled out. Labs in Table1. Measurement of immunoglobulins levels, ANCA and HIV serology were normal/negative. Diagnosis lymphopenic sarcoidosis's done. Prednisone $30 \mathrm{mg}$ per day had started and the disease improved with reduction of dyspnea, arthralgia and radiological improvement, although fatigue and arthralgia persisted. Hydroxychloroquine and methotrexate weren't tolerated and signs of glucocorticoids toxicity arised : cataract, osteopenia and obesity. Taper prednisone was attempt but disease activity returned. New labs in Table 1 . We returned prednisone to upper doses (30mg). Low CD4+ T Lymphocyte and concerns with glucocorticoids toxicity encouraged us to try a course of infliximab, a chimeric anti-TNF alpha antibody, one option of treatment for refractory sarcoidosis. Infliximab (5 mg/kg IV) was administered at 0,2 , and 6 weeks and maintenance dosing was continued every 8 weeks thereafter. After 8 months of treatment, the patient presented improvement of fatigue and arthralgia, reduction of pulmonary interstitial micronodules, satisfactory taper of prednisone $(6 \mathrm{mg} /$ day) and an increase of CD4 lymphocyte (Table1).

\section{CONCLUSION}

CD4 lymphopenia can be a distinct sarcoidosis phenotype that is particularly responsive to anti-TNF therapy. It is early to determine which treatment is best suited for each sarcoidosis phenotypes, but antitnf can be a strategy used. 